Apidologie, 1988, 19 (3), 245-254

\title{
EINFLUß VON KÄLTEEINBRÜCHEN AUF DIE FRÜHJAHRSENTWICKLUNG VON BIENENVÖLKERN (APIS MELLIFERA L.)
}

Jost H. DUSTMANN, Werner von der OHE

Niedersächsisches Landesinstitut für Bienenkunde, Wehlstr. 4a, D-3100 Celle

\section{ZUSAMMENFASSUNG}

Die erste Hälfte der Bienensaison eines Jahres ist in Niedersachsen in der Regel von mehreren Kältecinbrüchen gestört. Die vorliegenden Versuche zeigen einen deutlich negativen Einfluß derartiger Kälteeinbrüche auf die Entwicklung von Bienenvölkern, bedingt durch eine Unterbrechung der Pollenzufuhr. Die Brutentwicklung sowie die Entwicklung der Ammenbienen und damit die Versorgung der Larven werden beeinträchtigt. Die ungenügende Versorgung der Larven hat einen äußerst negativen Einfluß auf die aus ihnen hervorgehenden Imagines (mangelhafter Zustand der Ammenbienen). Die Störung bleibt nicht auf eine Generation beschränkt, vielmehr wird eine Kettenreaktion ausgelöst. Völkerverluste können die Folge sein.

Die durch mangelhafte Pollenzufuhr gestörte Proteinversorgung eines Volkes kann nicht nur anhand der Pollenvorräte, sondern muß auch anhand des physiologischen Zustandes und der intrakorporalen Proteinverteilung bei den Ammenbienen beurteilt werden.

\section{EINLEITUNG}

Der physiologische Zustand der Winterbienen sowie die generative Leistung der Königin sind entscheidend für die Überwinterung eines Bienenvolkes (HaydaK, 1970 ; Wille, 1974). Beide Parameter sind eng verknüpft mit dem Pollenversorgungsgrad (HaydaK, 1970 ; Maurizio, 1954). Die Überwinterungsfähigkeit ist nicht immer gegeben. Besonders im Jahr 1985 wurden hohe Völkerverluste beklagt (GNäDINGER, 1985). Derartige Frühjahrsprobleme in Bienenvölkern sind keine Neuheit (disappearing disease $=$ Frühjahrsschwindsucht). Sie sind vor allem auf mangelnde Pollenversorgung im Herbst und zeitigem Frühjahr zurückführen, wiederum erklärbar durch ein ungenügendes Trachtangebot (WILLE, 1973). Diese Situation allein kann aber nicht für die in Norddeutschland häufige verzögerte Frühjahrs- und Frühsommerentwicklung verantwortlich gemacht werden. Die Untersuchungen der vorliegenden Arbeit sollten zeigen, in wieweit die für den norddeutschen Raum typischen Kälteein- 
brüche (max. Tagestemperaturen für zwei und mehr aufeinanderfolgende Tage unter $12{ }^{\circ} \mathrm{C}=$ kein Flugwetter) im Zeitraum März - Juni als Ursache hierfür angesehen werden können. Man könnte die Auffassung vertreten, daß im Gegensatz zu der früher heimischen Rasse Apis mellifera mellifera die heute verbreitete Rasse Apis mellifera carnica mit ihrer genetisch verankerten sprunghaften Frühjahrsentwicklung (ZANDER/BöTrCHER, 1984) anfällig gegenüber Unterbrechungen der Pollenzufuhr durch Kälteeinbrüche ist.

\section{METHODIK UND MATERIAL}

Von März bis Juli wurden mit auf dem Institutsgelände stehenden Bienenvölkern Langzeitversuche durchgeführt. Gegenstand der Versuche war die natürliche, unbeeinflußte Entwicklung der Völker. Die Versuche des Jahres 1984 dienten zur Erarbeitung und Erprobung einer geeigneten Methodik. Die hier beschricbenen Ergebnisse beziehen sich ganz überwiegend auf den Versuch des Jahres 1985. 4 Völker wurden eingesetzt. Die Königinnen waren unterschiedlicher genetischer Abstammung. Die Erfassung des Witterungsverlaufes (Lufttemperatur, Luftfeuchtigkeit, Niederschlag, Windstärke und -richtung, Lichtintensität der Sonnenstrahlung) wurden mit der institutseigenen Wetterstation durchgeführt.

Ermittlung des Polleneintrages und der Bevorratung: Während der täglichen Zähltermine wurden die abfliegenden Bienen pro Minute und der prozentuale Anteil Pollensammlerinnen an den heimkehrenden Bienen ermittelt. Aus 3 Momentaufnahmen pro Zähltermin wurde der Mittelwert gebildet. Zwischenzeitlich wurden einige wenige Pollensammlerinnen abgefangen, um das Gewicht der Pollenhöschen und deren Herkunft zu bestimmen. Die Pollenvorratsflächen im Volk wurden bei der wöchentlichen Durchsicht mit der Zählrahmenmethode erfaßt (Maschenweite $4 \mathrm{~cm}^{2}$ ). Ermittlung der Brutentwicklung : Mit der Zählrahmenmethode wurden ebenso die Brutflächen der Völker wöchentlich erfaßt. Differenziert wurde offene Brut (Eier, Rundmaden) und verdeckelte Brut. Untersuchung der Ammenbienen : Wöchentlich wurden möglichst 10 eindeutige Ammenbienen entnommen, deren Untersuchung auf den 4 folgenden Parametern beruhte : "Gesamtproteingehalt der Hämolymphe " nach der Mikromethode von BradForD, 1976, "Vitellogenin-Titer der Hämolymphe » mit der Cellulose-Acetat-Elektrophorese nach Engels (1972), morphometrische Erfassung der « Acinibreite der Hypopharynxdrüsen " und "Proteinspeicherung der Fettkörper » (Einteilung in 5 Stufen).

\section{ERGEBNISSE}

Bei max. Tagestemperaturen im Schatten von $<12{ }^{\circ} \mathrm{C}$ bzw. $<16^{\circ} \mathrm{C}$ einhergehend mit Niederschlägen, waren keine flugaktiven Bienen zu verzeichnen (Abb. 1). Derartige Witterungserscheinungen hielten 2 oder mehr Tage an und unterschieden sich häufig drastisch von den vorangegangenen bzw. nachfolgenden Tagen, so daß der Begriff «Einbruch » gerechtfertigt erscheint. Diese witterungsbedingte Einstellung der Flugaktivität soll hier als « Kälteeinbruch » definiert werden.

Aus der Anzahl der abfliegenden Bienen pro Minute und dem Anteil der Pollensammlerinnen an heimkehrenden Bienen wurde die Flugaktivität der Pollensammlerinnen pro Momentaufnahme errechnet. Zu Beginn des Beobach- 
A

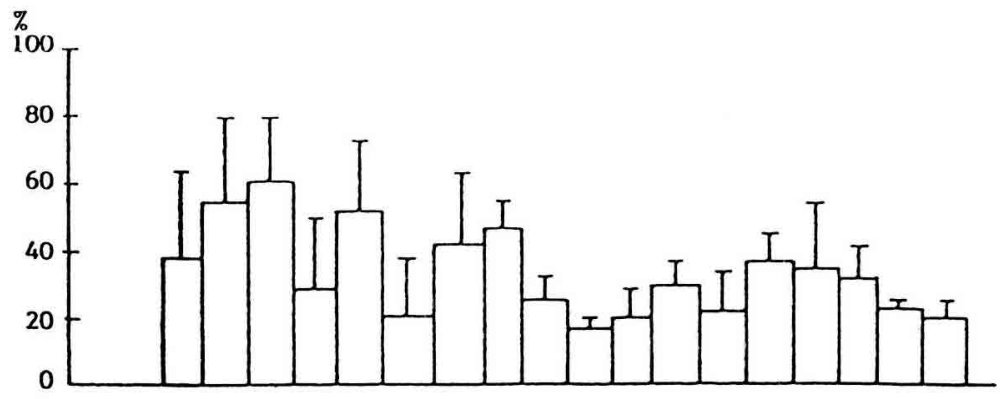

B
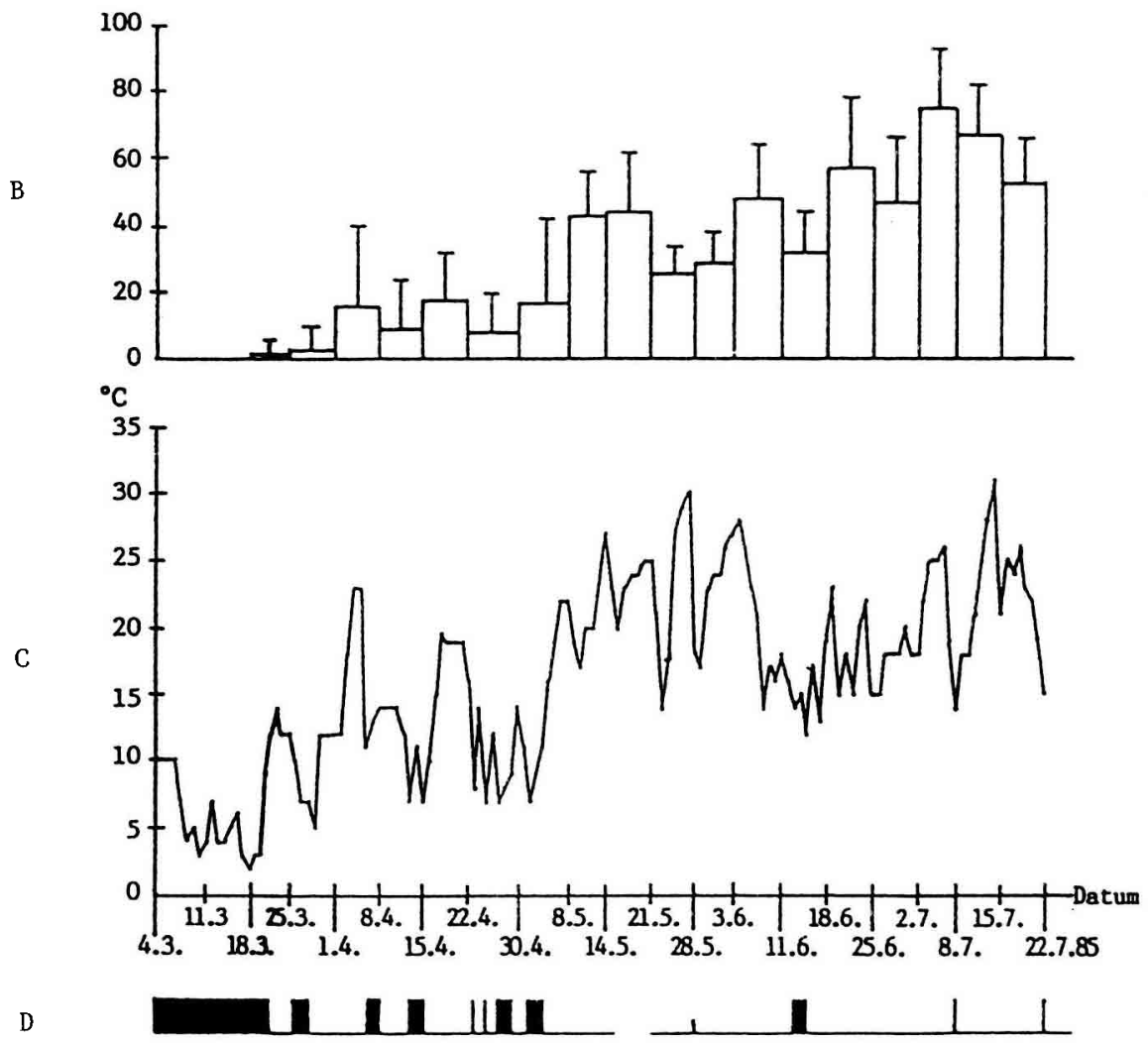

Aвв. 1. - Witterungsverlauf und Flugaktivität der Bienen im Zeitraum vom 4.3. bis 22.7.1985:

A) $\varnothing$ Anteil Pollensammlerinnen an flugaktiven Bienen insgesamt und

B) $\varnothing$ Anzahl abfliegender Bienen pro Minute zwischen 9.00 und 17.00 Uhr -

Mittelwerte und Standardabweichung von 4 Völkern über den Zeitraum einer Woche bzw. zwischen den Völker-Nachsicht-Terminen ;

C) max. Tagestemperatur in ${ }^{\circ} \mathrm{C}$; D) Tage ohne Bienenflug (markiert als schwarze Balken).

FIG. 1. - Meteorological data and flight activity of bees in the period 4.3. to 22.7.1985:

A) $\varnothing$ amount of pollen gathering bees in flight active bees and

B) $\varnothing$ number of flying off bees per minute during 9 a.m. and 5 p.m. - average of 4 colonies during one week respectively between the weekly inspection ;

C) max. daytime temperature in ${ }^{\circ} \mathrm{C}$; D) day without flight activity (indicated as black bars). 
tungszeitraumes war der Anteil der Pollensammlerinnen an den sammelnden Bienen hoch und nahm über die Wochen bis zum Sommer ab. Sammelgutausfälle durch Kälteeinbrüche wurden zunächst beim Pollen kompensiert.

Die Flugaktivität der Pollensammlerinnen während eines Zeitpunktes war bei den Völkern unterschiedlich hoch, die Veränderungstendenzen über die Zeit hinweg jedoch gleich. Die Flugaktivität der Pollensammlerinnen und damit die Quantität des Polleneintrages spiegelt sich in den Pollenvorratsflächen wider. Die Brutentwicklung (offene Brut) läuft bis auf wenige Ausnahmen synchron mit der Veränderung der Flugaktivität der Pollensammlerinnen. Es liegt eine positive Korrelation von $r=+0,889$ vor. Ebenso wie bei Flugaktivität und Pollenvorratsfläche lagen auch bei den Brutflächen Differenzen zwischen den verschiedenen Völkern vor, während die Entwicklungstendenzen bis auf wenige Ausnahmen gleich waren. Auffällig erschien eine deutlich verkleinerte Fläche mit verdeckelter Brut in Relation zu der Fläche mit offener Brut (vgl. Abb. 2).

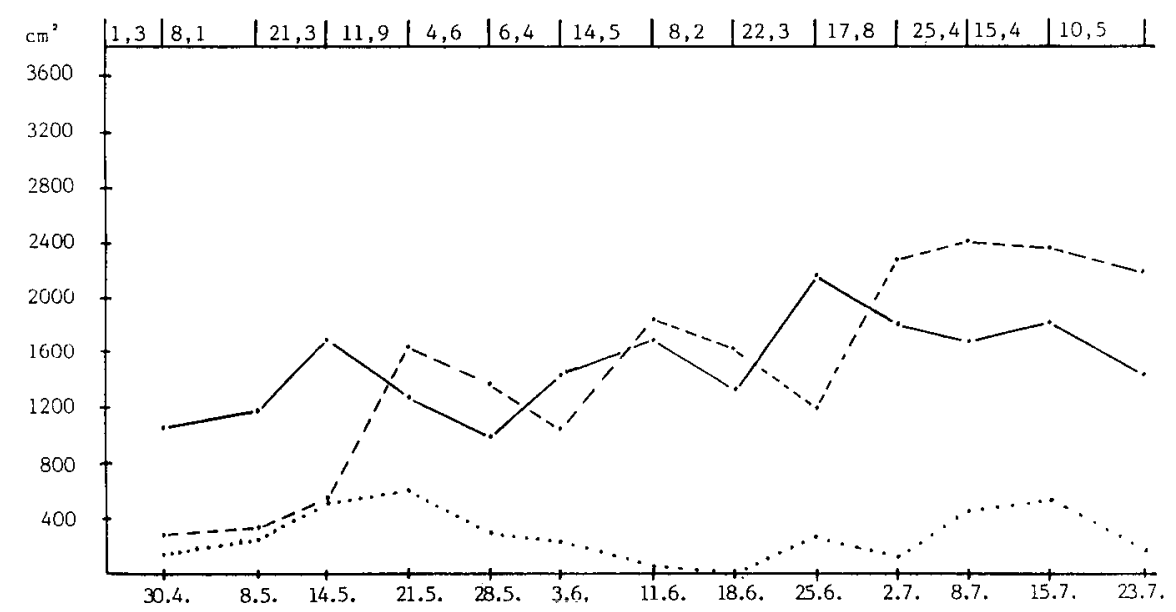

Aвв. 2. - Brut- und Pollenbevorratungs-Flächen in $\mathrm{cm}^{2}$ (Mittelwerte von 4 Völkern) :

offene Brut (-), verdeckelte Brut (--), Bevorratung (...) ; Zahlen oberhalb der Parallelen zur Abszisse : $\varnothing$ Flugaktivität der Pollensammlerinnen pro Minute in den Zeiträumen zwischen den Völkerkontrollen.

Fig. 2. - Area of brood nest and pollen store in $\mathrm{cm}^{2}$ (average of 4 colonies) :

unsealed brood $(-)$, sealed brood $(--)$, stock $(\ldots$.$) ; numbers at the top : \varnothing$ flight activity of pollen gathering bees per minute during the periods between the weekly colony inspections.

Entwicklung der Ammenbienen : Die Anzahl der für die Untersuchungen zur Verfügung stehenden Ammenbienen pro Volk bzw. Nachschautermin schwankte erheblich (vergl. Abb. 3). Große Differenzen zwischen den Unter- 

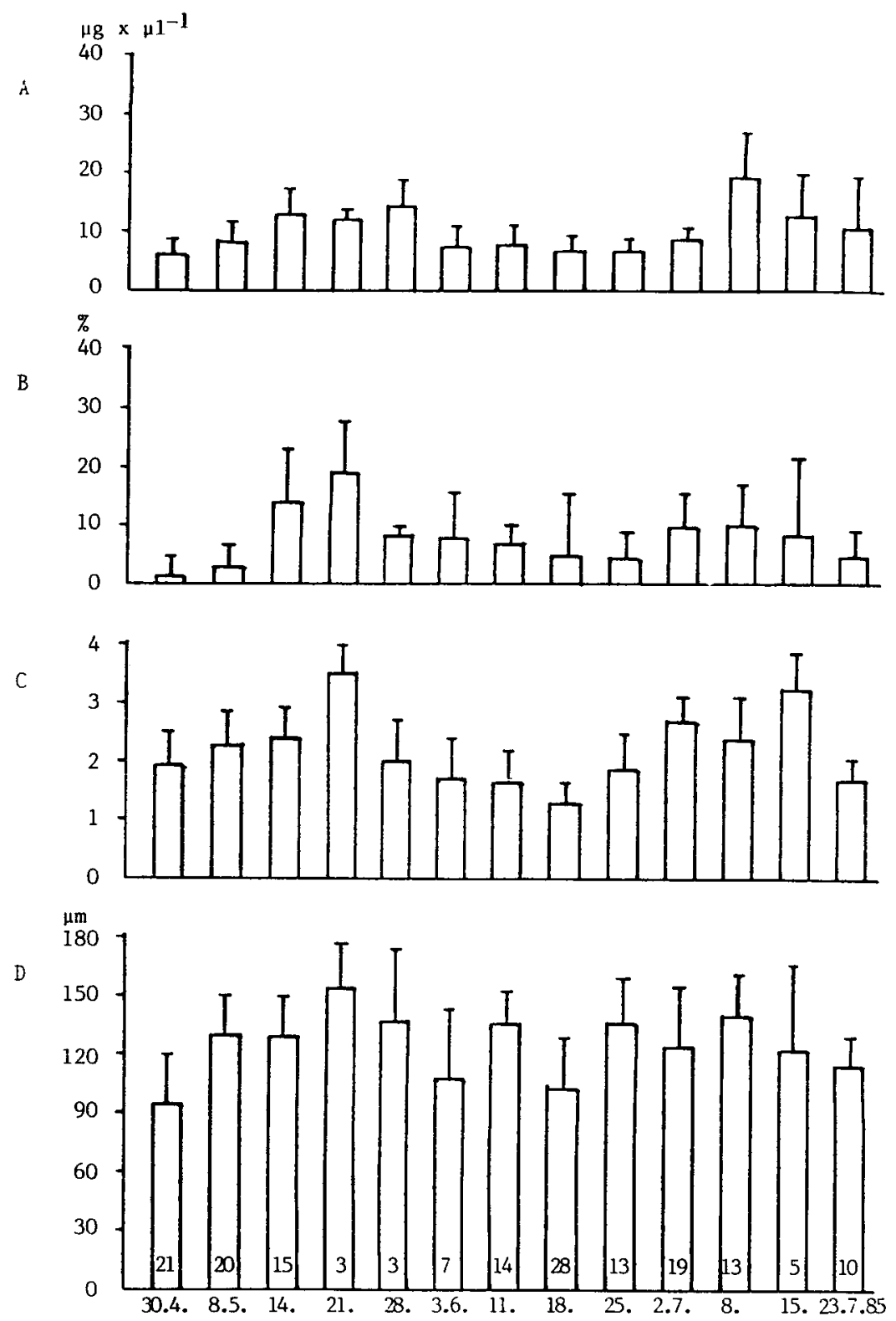

Aвв. 3. - Entwicklungsgrad der Ammen, die bei den Völker-Nachsichten aus den 4 Völkern entnommen wurden:

A) $\varnothing$ Proteingehalt $\left(\mu \mathrm{g} \times \mu^{-1}\right)$ und

B) $\varnothing$ Vitellogenin-Titer (Vtg) in \% des Gesamtproteingehaltes der Hämolymphe pro Biene,

2) $\varnothing$ Entwicklungsgrad des Fettkörpers (FK) und D) $\varnothing$ Acini-Breite der Hypopharynxdrüsen (HPD) in $\mu \mathrm{m}$; Mittelwert je Entnahmetermin mit Standardabweichung ;

Anzahl der Ammen je Termin in den Säulen der unteren Graphik.

FIG. 3. - Development of nurse bees getting out of the 4 colonies at the weekly inspections :

A) $\varnothing$ protein- and B) vitellogenin content of the haemolymph per bee,

C) $\varnothing$ development of fatbody and D) acini width of the hypopharyngeal glands ; quantity of bees per set point are represented in the columns. 
suchungsterminen wiesen der Gesamtproteingehalt und der Vitellogenin-Titer der Hämolymphe auf. Ebenso zeigte der Entwicklungsgrad der Fettkörper zwischen den Nachschauterminen relativ große Unterschiede, während die Hypopharynxdrüsen einen relativ hohen und etwas gleichmäßigeren Entwicklungslevel aufwiesen. Ein niedriger Entwicklungsgrad trat besonders nach längeren Phasen schlechter Witterung auf.

\section{DISKUSSION}

Das häufige Auftreten von Kälteeinbrüchen im Zeitraum März bis Juni in den Versuchsjahren 1984 und 1985 erweist sich für den norddeutschen Raum als typisch, wie die Klimadaten der vergangenen 11 Jahre zeigen (Klimadaten 1975-1981 aus Aufzeichnungen des deutschen Wetterdienstes - Wetterämter Hannover-Langenhagen und Bremen ; 1982-1985 Wetterstation des Instituts). Die vorliegenden Versuche zeigten deutlich negativen Einfluß der Kälteeinbrüche auf die Sammelaktivität der Bienen. Die Flugaktivität ist positiv korreliert mit der Höhe der max. Tagestemperatur $(r=+0,837)$. Die Aktivität der Pollensammlerinnen sowie Größe und Gewicht der Pollenhöschen sind von der Witterung abhängig.

Eine positive Korrelation zwischen der Aktivität der Pollensammlerinnen und der Pollenvorratsfläche ließ sich nicht errechnen. Ursache hierfür ist wahrscheinlich darin zu suchen, daß zum Zeitpunkt des gesteigerten Polleneintrages auch eine große Fläche an offener Brut vorliegt, hieraus resultiert ein erhöhter Pollenbedarf, so daß die Ausdehnung der Vorratsflächen verhindert wird. Eine positive Korrelation wurde zwischen Polleneintrag und Brutentwicklung nachgewiesen. Die Unterbrechung der Pollenzufuhr führte zu Störungen in der Brutentwicklung. Während WILLE (1985) einen direkten Einfluß der Pollenversorgung auf die Brutentwicklung bestreitet und den genetischen Einfluß betont, weist außer uns auch Doull (1975) auf eine direkte positive Korrelation zwischen Pollenangebot und Brutentwicklung hin. RuTTNER/RuTTNER (1976) sehen ebenfalls in der Umwelt einen größeren Einflußfaktor auf die Brutentwicklung von Bienenvölkern als in der Genetik. Eine Steigerung des Polleneintrages stimuliert die Königin zur Eiablage, eine Verminderung des Polleneintrages hat den umgekehrten Effekt und kann bei einem zu geringen Pollenangebot im Volk über das Regulativ «Stockhygiene " bzw. "Kannibalismus » zusätzlich die Brutentwicklung stören (WEISs, 1984).

Kannibalismus hat wahrscheinlich in allen Versuchsvölkern stattgefunden. Die Untersuchung der Ammenbienen zeigte eine Beeinträchtigung ihrer Entwicklung durch Pollenmangel. Die Hypopharynxdrüsen der Ammen werden bei vorhandener Brut bevorzugt mit Protein versorgt. Die Anwesenheit von Brut 
hat nach Brouwers $(1982,1983)$ Einfluß auf die Größe und ProteinsyntheseAktivität der Hypopharynxdrüsen. Der Fettkörper dient dagegen vor allem als Metabolismus-Zentrale, erst bei einem Überangebot kommt es hier zu einer Proteinspeicherung. Trotz der Dominanz der Hypopharynxdrüsen in der innerkörperlichen Proteinversorgung der Ammen, hatten extreme Verminderungen der Pollenzufuhr auch einen negativen Einfluß auf die Drüsenentwicklung.

Die Bienen können ein im larvalen Fettkörper gespeichertes Überangebot an Nährstoffen über die Metamorphose in das imaginale Stadium transferieren. Nach den vorliegenden Ergebnissen ist für die Entwicklung der Ammen nicht nur die Proteinversorgung während der Ammenzeit, sondern auch die während der Larvenzeit von größter Bedeutung. Ein Beispiel hierzu : die Hypopharynxdrüsen der Ammen von 2.7.85 in Volk 12 sind trotz einer Vielzahl an zu versorgender Brut und hohem Polleneintrag der Vorwoche relativ schwach entwickelt. Während des Rundmadenstadiums dieser Ammen war eine erhebliche Schmälerung des Polleneintrages zu verzeichnen.

Verminderter Polleneintrag wirkt sich negativ auf die Brutentwicklung aus. Die reduzierte Population der nächsten Generation von Imagines kann dementsprechend weniger Ammenbienen zu Verfügung stellen, die wiederum gezwungen sind, pro Individuum eine größere Anzahl Larven zu versorgen. EISCHEN et al. (1983) konnten bei einem « Mehr » an Larven pro Amme eine schlechtere Entwicklung der aus diesen Larven hervorgehenden Imagines nachweisen. De Groor (1953/54) fand ebenfalls diese Abhängigkeit des Entwicklungsgrades von der Relation Larven zu Ammen.

Damit liegt in der Proteinversorgung und Populationsdynamik ein Generationsproblem vor. Kälteeinbrüche beeinflussen die Populationsdynamik unter Umständen über mehrere Generationen hinweg negativ. Eine häufige Aufeinanderfolge von Kälteeinbrüchen, wie sie für Niedersachsen vorliegt, kann demnach katastrophale Auswirkungen haben. Die kumulative Wirkung von negativen Faktoren über einige Generationen dürfte sehr wahrscheinlich die Überlebenschance der Völker drastisch mindern.

Aus den vorliegenden Ergebnissen kann man bereits folgenden Schluß ziehen : Beim Auftreten von Kälteeinbrüchen erscheint die Gabe von Pollenersatzstoffen unerläßlich. Allerdings kann nur mit einem bienengerechten Pollenersatzstoff bei Fütterung außerhalb der Beute die Kontinuität der Völker gesichert werden (von der OHE, 1986). Es erscheint sinnvoll, zur Erhärtung der vorliegenden Ergebnisse weitere Langzeitversuche auch in anderen Biotopen durchzuführen. 
Die vorliegende Arbeit wurde durch Sonderforschungsmittel des Landes Niedersachsen finanziert. Hierfür danken wir dem Nieders. Minister für Ernährung, Landwirtschaft und Forsten.

\author{
RÉSUMÉ \\ INFLUENCE DES COUPS DE FROID SUR LE DÉVELOPPEMENT PRINTANIER \\ DES COLONIES D'ABEILLES (APIS MELLIFICA L.)
}

Les relevés météorologiques des 10 dernières années montrent que le printemps en Basse-Saxe (R.F.A.) est perturbé par des coups de froid. Les coups de froid sont des périodes de deux ou plusieurs jours au cours desquelles les abeilles ne présentent pas d'activité de vol (température maximale de la journée $<12{ }^{\circ} \mathrm{C}$ ou $<16^{\circ} \mathrm{C}$ avec pluie). Afin d'en étudier l'influence sur le développement des colonies, on a enregistré, au cours d'expériences de longue durée, les conditions météorologiques et suivi le développement des colonies : activité de vol - principalement des butineuses de pollen -, élevage du couvain, stockage de pollen, développement des nourrices (teneur en protéines et en vitellogénine de l'hémolymphe, glandes hypopharyngiennes, corps gras).

Les résultats montrent que la température maximale de la journée est directement corrélée avec l'activité de vol des abeilles. Les coups de froid inhibent l'activité de vol et conduisent par conséquent à une interruption de l'approvisionnement en pollen. L'activité des butineuses de pollen est directement corrélée avec le taux de ponte de la reine. Une faible activité de récolte a aussi une action négative sur l'élevage du couvain par cannibalisme de couvain. La population de la génération suivante étant réduite fournit donc moins de nourrices. Pour cette raison une nourrice doit s'occuper d'un plus grand nombre de larves. Plus de larves par nourrice signifie un approvisionnement moindre pour chacune d'elle.

Le faible approvisionnement en pollen dû aux coups de froid influence le développement du couvain et des nourrices. Ils sont tous deux incapables d'avoir leurs besoins en nutriments assurés. Il s'ensuit que les nourrices - particulièrement leurs glandes hypopharyngiennes - sont mal développées. Elles ne peuvent donc pas fournir suffisamment de gelée d'ouvrière aux larves. Ceci agit négativement sur le stade imaginal de ces abeilles. Elles ne peuvent devenir de bonnes nourrices que si un approvisionnement suffisant en pollen est assuré au cours de leur activité de nourrice. Il en résulte un "conflit de générations "

\title{
SUMMARY
}

\section{EFFECT OF COLD SNAPS ON THE BUILD UP OF HONEYBEE COLONIES (APIS MELLIFERA L.) IN SPRINGTIME}

Meteorological data of the last 10 years show that in Lower-Saxony (F.R.G.) springtime is disturbed by several cold snaps. Cold snaps are defined as two or more successive days without flight activity of honeybees (max. daytime temperature $<12{ }^{\circ} \mathrm{C}$ or $<16{ }^{\circ} \mathrm{C}$ with rain). In long-term experiments meteorological conditions were recorded and bee-colonies were examined to investigate the influence of cold snaps. Factors relevant to flight activity - especially of pollen gathering bees - were examined. These included : brood rearing, pollen intake and store, development of nurse bees (protein and vitellogenin content of haemolymph, hypopharyngeal glands and fat body). The results show that the max. daytime temperature ist directly correlated with the flight activity of honeybees. Cold snaps prevent flight activity and by that lead to an interruption of pollen intake. Activity of pollen gathering bees is directly correlated to the egg laying rate of the queen. Low gathering activity has also a negative effect 
of brood rearing, by increasing brood cannibalism. Accordingly, the reduced population of the next generation means there are less nurse bees present. Due to this, one nurse bee has to supply a greater number of larvae. More larvae per nurse bee means a poor supply of food to the individual larvae.

The poor pollen supply resulting from cold snaps influences the development of brood and of nurse bees. Both are unable to get their requirements of nutrients. So the nurse bees - especially their hypopharyngeal glands - are not adequately developed. Nurse bees whose hypopharyngeal glands are not adequately developed, due to an insufficient supply of pollen (protein) during larvae and early imaginal stages, are not able to adequately supply open brood with worker jelly. Thus, an adequate supply of nutrients to the imaginal stages is important. However, to become optimal nurse bees, adults also require an adequate supply of pollen during their nurse activity. The result is a generation conflict ».

\section{LITERATURVERZEICHNIS}

BRADFORD M.M., 1976. - A rapid and sensitive method for the quantitation of microgram quantities of protein utilizing the principle of protein-dye binding. Anal. Biochem. 72, 248-254.

Brouwers E.V.M., 1982. - Measurement of hypopharyngeal gland activity in the honeybee. J. Apic. Res., 21, 193-198.

Brouwers E.V.M., 1983. - Activation of the hypopharyngeal glands of the honeybees in winter. $J$. Apic. Res., 22, 137-141.

Doull K.M., 1975. - Pollen supplements Part I-III. Am. Bee J., 115, 14-15, 54-55, 88-89, 99.

Eischen F.A., Rothenbuhler W.C., Kulincevic J.M., 1983. - Brood rearing associated with a range of worker-larva ratios in the honeybee. $J$. Apic. Res., 22, 163-168.

ENGELS W., 1972. - Quantitative Untersuchungen zum Dotterprotein-Haushalt der Honigbiene (Apis mellifera). Wilhelm Roux'Arch., 171, 55-86.

GnÄdinger F., 1985. - Auswinterungsverluste bei Bienenvölkern in Baden. Allg. dtsch. Imkerztg., 19 , 297-299.

Groot A.P. de, 1953/54. - Protein and amino acid requirements of the honeybee (Apis mellifica L.). Physiol. Comp. et Oecol., 3, 197-285.

Haydak M.H., 1970. - Honey bee nutrition. Annu. Rev. Entomol., 15, 143-156.

Maurizio A., 1954. - Pollenernährung und Lebensvorgänge bei der Honigbiene (Apis mellifica L.). Landwirtsch. Jahrb. Schweiz, 68, 115-193.

OHE W. von der, 1986. - Proteinpräparate in vergleichenden ernährungs- und verhaltensphysiologischen Untersuchungen an Honigbienen (Apis mellifera L.) im Hinblick auf die Verwendung als bienengerechter Pollenersatzstoff. Dissertation, Universität Hannover.

Ruttner F, RutTner H., 1976. - Die Spätsommerbrut bei Völkern verschiedener Abstammung und ihre Abhängigkeit von Umweltbedingungen. Allg. dtsch. Imkerztg., 10, 417-421.

Weiss K., 1984. - Regulierung des Proteinhaushaltes im Bienenvolk (Apis mellifica L.) durch Brutkannibalismus. Apidologie, 15, 339-354.

Wille H., 1973. - Fragen um die Pollenversorgung. Schweiz. Bienenztg. 96, 572-579.

Wille H., 1974. - Massenwechsel des Bienenvolkes, Teil I-III. Schweiz. Bienenztg, 97, 304-316, 369$374,420-425$.

Wille H., 1985, - Überlebensstrategien des Bienenvolkes. Bienenwelt, 27, 169-182.

Zander E., Börtcher F.K., 1984. - Krankheiten der Biene. 7. neubearb. und erweit. Auflage, Ulmer, Stuttgart. 